\title{
The Optimization Ordering Model for Intuitionistic Fuzzy Preference Relations with Utility Functions
}

\author{
Zaiwu Gonga,*, Ning Zhang ${ }^{\mathrm{b}}$, Francisco Chiclana ${ }^{\mathrm{c}}$ \\ ${ }^{a}$ School of Management Science and Engineering, Nanjing University of Information Science and Technology, Nanjing \\ 210044, China; School of Business, Linyi University, Linyi 276000, China \\ ${ }^{b}$ School of Management Science and Engineering, Nanjing University of Information Science and Technology, Nanjing \\ 210044, China \\ ${ }^{c}$ Institute of Artificial Intelligence, School of Computer Science and Informatics, De Montfort University, Leicester LE1 \\ $9 B H, U K$ \\ Dept. of Computer Science and Artificial Intelligence, University of Granada, Granada, Spain
}

\begin{abstract}
Intuitionistic fuzzy sets describe information from the three aspects of membership degree, nonmembership degree and hesitation degree, which has more practical significance when uncertainty pervades qualitative decision problems. In this paper, we investigate the problem of ranking intuitionistic fuzzy preference relations (IFPRs) based on various non-linear utility functions. First, we transform IFPRs into their isomorphic interval-value fuzzy preference relations (IVFPRs), and utilise non-linear utility functions, such as parabolic, S-shaped, and hyperbolic absolute risk aversion, to fit the true value of a decision-maker's judgement. Ultimately, the optimization ordering models for the membership and non-membership of IVFPRs based on utility function are constructed, with objective function aiming at minimizing the distance deviation between the multiplicative consistency ideal judgment and the actual judgment, represented by utility function, subject to the decision-maker's utility constraints. The proposed models ensure that more factual and optimal ranking of alternative is acquired, avoiding information distortion caused by the operations of intervals. Second, by introducing a non-Archimedean infinitesimal, we establish the optimization ordering model for IFPRs with the priority of utility or deviation, which realises the goal of prioritising solutions under multiobjective programming. Subsequently, we verify that a close connection exists between the ranking for membership and non-membership degree IVFPRs. Comparison analyses with existing approaches are summarized to demonstrate that the proposed models have advantage in dealing with group decision making problems with IFPRs.
\end{abstract}

Keywords: Intuitionistic fuzzy preference relation, Utility Function, Ranking, Multiplicative consistency, Non-archimedean infinitesimal.

\footnotetext{
${ }^{*}$ Corresponding author

Email addresses: zwgong26@163.com (Zaiwu Gong), lyguangzhiying@163.com (Ning Zhang), chiclana@dmu.ac.uk (Francisco Chiclana)
} 


\section{Introduction}

In multi-attribute decision making, decision makers (DMs) often construct a judgment matrix or preference relation (PR) by pairwise comparison for optimal alternative selection. Different types of PRs have been proposed in multi-attribute decision making both in a crisp context (reciprocal PRs) and in a fuzzy context (fuzzy reciprocal PRs). In this last one it is worth mentioning the following: interval-value fuzzy PRs (IVFPRs) [1-5], intuitionistic fuzzy PR (IFPR) [6-8], hesitant fuzzy PR (FPR) [9-11], and linguistic fuzzy PR [12-15]. Due to the isomorphism between intuitionistic fuzzy sets and interval fuzzy sets, interval operations methods are used to solve decision problems with IFPRs [16-21]. Moreover, IFPRs describe a DM's uncertain judgment preference information using the three aspects of membership degree, non-membership degree and hesitation degree, which has more practical significance when dealing with multi-attribute group decision making (GDM) with uncertainty [22-27].

Consistency (multiplicative consistency and additive consistency) cannot only guarantee the reliability and accuracy of alternative ranking, but may also help in obtaining an optimal consensus decision. Behret [28] proposed IFPRs models based on additive consistency and multiplicative consistency. Liao and $\mathrm{Xu}$ [29] explored the consistency between IFPRs and group intuitionistic fuzzy analytic hierarchy process (AHP). Wan et al. [30] established an optimization model based on consistency to solve a GDM problem with interval IFPRs, while Xu et al. [31] developed a mathematical programming method to improve the degree of individual IFPR consistency. In order to achieve consensus within a group of experts, Chu et al. [32] proposed an algorithm for IFPRs GDM satisfying additive consistency. Based on ranking consistency and multiplicative consistency, two GDM methods were developed by Jin et al. [33] to obtain IFPRs' optimal weights. Based on multiplicative consistency property, Meng et al. [6] established 0-1 mixed programming models to solve the GDM problem with IFPRs, while on additive consistency property Wan et al. [7] adopted a likelihood comparison algorithm and realised the optimal sequence ordering for GDM problem with IFPRs.

A utility function [34] refers to a function that measures the extent to which consumers are satisfied from the consumption of an established commodity combination $[35,36]$. In the framework of expected utility theory [37], if the expected utility value for an alternative $x^{\prime}$ is higher than the expected utility value for another alternative $x^{\prime \prime}$, then the alternative $x^{\prime}$ is considered better than the alternative $x^{\prime \prime}$. Meanwhile, PR reflects the preference intensity of the comparison between each two alternatives in the alternatives set, and consequently we can assume that each of the alternative's utility value corresponds to their PRs. As DMs tend to show different preference attitudes and satisfaction levels when giving opinions (i.e., defined as utility), and so the change of their utility varies with respect to the value in an interval opinion can be expressed using different utility functions. In 1968, Borch [38] put forward the concept of goal-based utility, which has been further generalised by Castagnoli and Calzi [39], Bordley and Kirkwood [40], Tsetlin and Winkler [41] and Durbach [42], and since then utility functions have 
been well extended and applied in GDM problems. Chang [43] employed the implementation level of a utility function to replace the expected level of a multi-objective problem's scalar value, and afterwards in [44] studied the application of a binary utility function in multi-objective fractional programming. Based on multi-attribute utility theory (MAUT), Musal et al. [45] presented a utility measured model combining MAUT and Bayesian statistics. In specific applications, complex utility function research has been successfully applied to portfolio selection [46], strategic choice [47], double auction [48], the interpretation of agricultural economics phenomena [49] and other decision-making issues related to behaviour and psychology. For instance, Ho et al. [50] proposed fuzzy goal programming with Sshaped utility functions for house selection via the internet by considering homebuyers' attitudes to risk. Kang and Ye [51] investigated three-country advantageous redistribution with two Cobb-Douglas utility functions and a constant elasticity of substitution (CES) utility function, while Breinbjerg [52] constructed an application case with a non-linear utility function to achieve equilibrium arrival times to queues with general service times.

Each value of a DM in interval judgment actually represents a utility of a DM. A utility function is utilised to fit the value of a DM in interval judgment, which can simulate the DM's psychological preference more authentically. The information of intuitionistic fuzzy numbers is handled with intervals due to their aforementioned isomorphic relationship. The acquisition of intervals by DMs is often expressed as a preference, which can be simulated using a utility function: each value in interval judgment corresponds to a utility value. In other words, a utility value always corresponds to a certain value in interval judgment (inverse function of the utility value). By constructing an appropriate utility function, the true value of a DM in its interval judgment can be fitted more truthfully. As different DMs can give different utility functions, we explore the ordering modelling for IFPRs based on various utility functions. To realise the generality of utility functions in the ordering modelling application, this paper assumes that various utility functions are invertible in DMs' interval opinions, and proposes the following research methodology:

- constructing IVFPRs equivalent to IFPRs, by using their isomorphism;

- selecting the matching utility function for the IFPR given by DM;

- constructing the ordering model for the membership and non-membership degree IVFPRs based on various utility functions, and calculating and analysing their optimal solutions;

- optimization ordering model for IFPRs under the priority of utility or deviation is proposed by introducing a non-Archimedean infinitesimal.

The remainder of this paper is organised as follows. Section 2 introduces several common non-linear utility functions, and definitions of IFPRs and IVFPRs. In Section 3, we propose modeling steps and the optimization ordering models for IFPRs based on various utility functions. In addition, the links 
between the optimization ordering models for the membership degree and non-membership degree of IVFPRs based on various utility functions are established. Section 4 introduces the non-Archimedean infinitesimal, and constructs the optimization ordering model for IFPRs under the priority of utility or deviation. Based on the proposed ranking models, Section 5 presents a calculation example and analysis combining the suppliers selection case and the proposed ordering model. Finally, Section 6 is conclusions.

\section{Preliminaries}

\subsection{Utility function}

\subsubsection{Fundamental assumptions}

- In GDM, it is assumed that the DMs' opinions are intervals for more confidently expressing attitudes. Let the opinion interval of individual $d_{i}, i \in M=\{1,2, \ldots, m\}$, be $o_{i}=\left[o_{i}^{L}, o_{i}^{U}\right]$.

- Any decision value in an interval corresponds to a utility value of the DM. Given a judgment interval $o_{i}=\left[o_{i}^{L}, o_{i}^{U}\right]$, any $x \in o_{i}$ corresponds to a utility value $u(x)$ of DM $d_{i}$.

Common utility functions include risk-seeking, risk-averse (see Fig. 1).
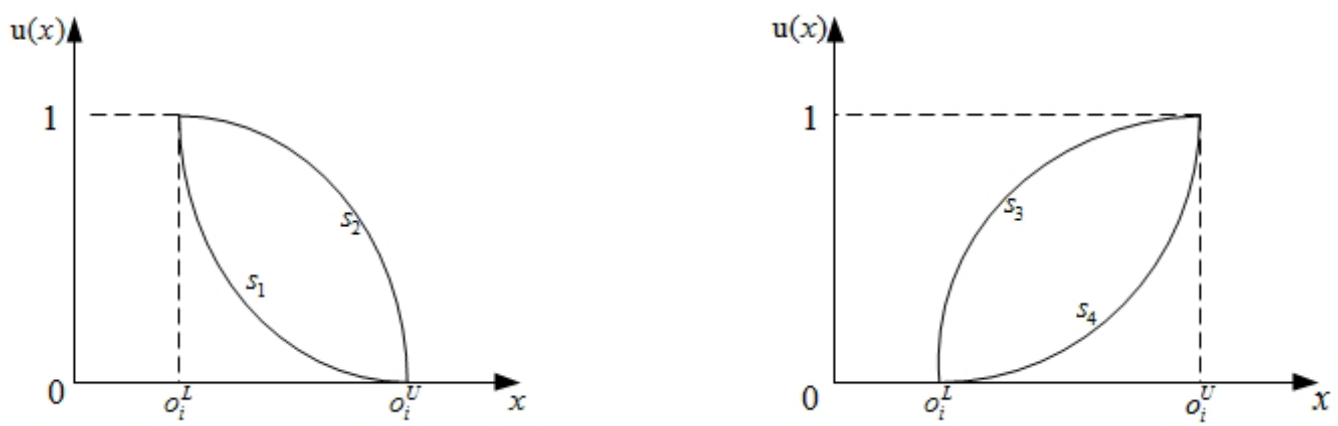

Fig. 1: Risk-seeking and risk-averse utility functions

In Fig. 1, the horizontal axis shows the opinion value $x$ of individual $d_{i}, x \in\left[o_{i}^{L}, o_{i}^{U}\right]$, and the vertical axis shows a utility value $u(x)$ of the DM's opinion, $0 \leq u(x) \leq 1$ :

- the utility function of curve $s_{1}$ is $u_{1}(x), x \in\left[o_{i}^{L}, o_{i}^{U}\right]$, satisfying $u_{1}^{\prime}(x) \leq 0, u_{1}^{\prime \prime}(x) \geq 0$ in the opinion interval;

- the utility function of curve $s_{2}$ is $u_{2}(x), x \in\left[o_{i}^{L}, o_{i}^{U}\right]$, satisfying $u_{2}^{\prime}(x) \leq 0, u_{2}^{\prime \prime}(x) \leq 0$ in the opinion interval;

- the utility function of curve $s_{3}$ is $u_{3}(x), x \in\left[o_{i}^{L}, o_{i}^{U}\right]$, satisfying $u_{3}^{\prime}(x) \geq 0, u_{3}^{\prime \prime}(x) \leq 0$ in the opinion interval;

- the utility function of curve $s_{4}$ is $u_{4}(x), x \in\left[o_{i}^{L}, o_{i}^{U}\right]$, satisfying $u_{4}^{\prime}(x) \geq 0, u_{4}^{\prime \prime}(x) \geq 0$ in the opinion interval. 
The above utility functions satisfy the following properties:

(i) the marginal utility values of curves $s_{1}$ and $s_{4}$ increase with the increase of the DM's opinion, and then utility function $u_{1}(x)$ and $u_{4}(x)$ are called risk-seeking functions;

(ii) in contrast, the marginal utility values of curves $s_{2}$ and $s_{3}$ decrease with the increase of the DM's opinion, and then utility function $u_{2}(x)$ and $u_{3}(x)$ are called risk-averse functions.

In complex GDM, the utility function of the DM's opinion is not necessarily simply risk-seeking or risk-averse, and it could be a combination of these two forms. Fig. 2 illustrates one such utility functions, the S-shaped utility function introduced by Kahneman and Tversky [53] in 1979, where the utility in opinion sub-interval $\left[o_{i}^{L}, o_{i}^{M}\right]$ satisfies $u^{\prime}(x) \geq 0, u^{\prime \prime}(x) \geq 0$, i.e. it is risk-seeking type; while the utility in opinion sub-interval $\left[o_{i}^{M}, o_{i}^{U}\right]$ satisfies $u^{\prime}(x) \geq 0, u^{\prime \prime}(x) \leq 0$, i.e. it is risk-averse type.

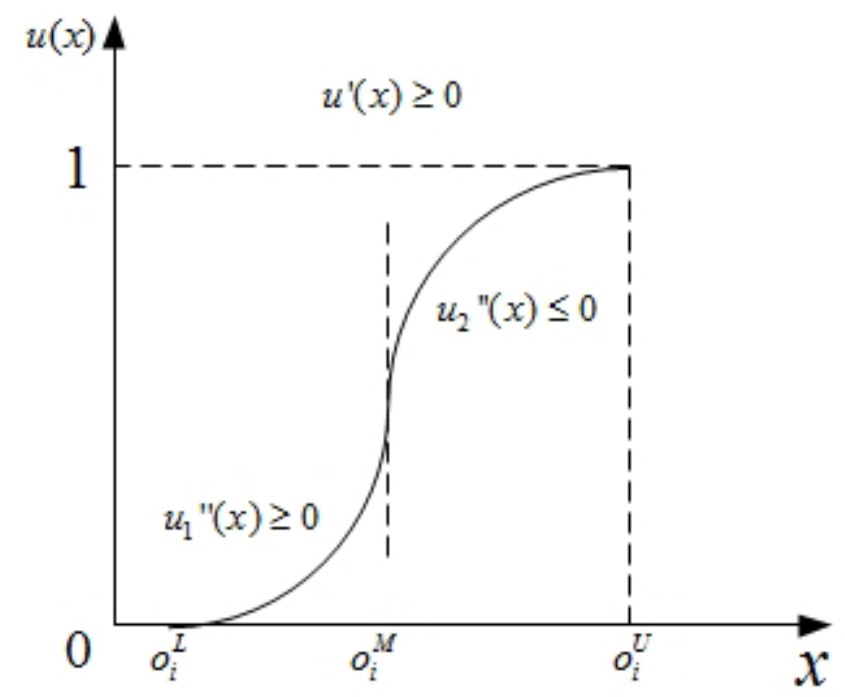

Fig. 2: S-shaped utility function

\subsubsection{Three specific non-linear utility functions}

We can establish myriads of utility functions for DMs' risk attitudes and utility preferences. Nevertheless, we discuss three specific non-linear utility functions: parabolic utility functions [54], S-shaped utility functions based on a sine function [54], and hyperbolic absolute risk aversion (HARA) utility functions $[47,55]$.

(i) Parabolic utility function. Let $x$ denote the interval opinion of DM $i, x \in\left[b_{i 1}, b_{i 2}\right]$, and let $u(x)$ denote the utility of DM $i$ 's opinion, $0 \leq u(x) \leq 1$. Assume that $b_{i 1}, b_{i 2}$ are known critical points (reference points) within the range of DM $i$ 's opinion. Parabolic utility functions are of two types (shown in Fig. 3):

1. left-skewed parabolic utility function: the smaller the DM's opinion values are, the higher their utility; 
2. right-skewed parabolic utility function: the larger DM's opinion, the higher the utility.

Expressions of DM's opinion corresponding to these type of parabolic utility functions are presented in Table 1.

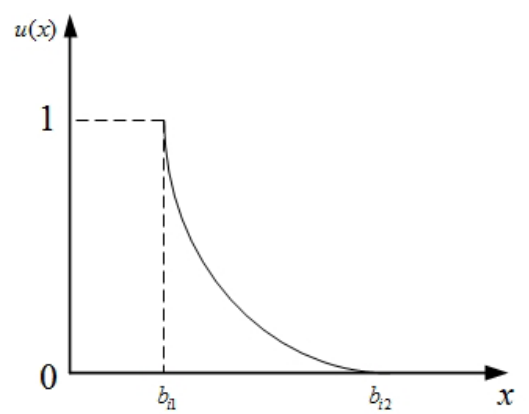

3.1 Left-skewed parabolic utility function

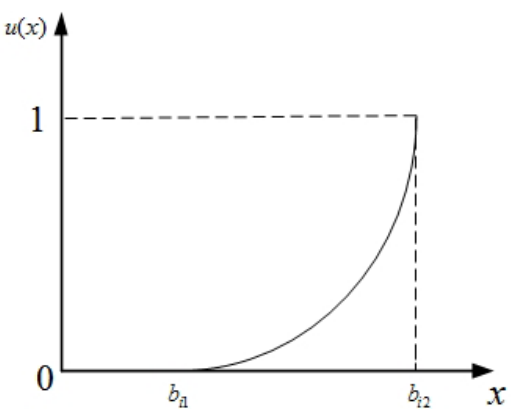

3.2 Right-skewed parabolic utility function

Fig. 3: Left-skewed and right-skewed parabolic utility functions

Table 1: Parabolic utility functions

\begin{tabular}{|c|c|c|}
\hline & Left-skewed parabolic utility function & Right-skewed parabolic utility function \\
\hline Utility function & $u(x)=\left(\frac{b_{i 2}-x}{b_{i 2}-b_{i 1}}\right)^{2}, b_{i 1} \leq x<b_{i 2}$ & $u(x)=\left(\frac{x-b_{i 1}}{b_{i 2}-b_{i 1}}\right)^{2}, b_{i 1} \leq x<b_{i 2}$ \\
\hline DM's opinion & $x=b_{i 2}-\sqrt{u(x)}\left(b_{i 2}-b_{i 1}\right), b_{i 1} \leq x<b_{i 2}$ & $x=\sqrt{u(x)}\left(b_{i 2}-b_{i 1}\right)+b_{i 1}, b_{i 1} \leq x<b_{i 2}$ \\
\hline
\end{tabular}

(ii) S-shaped utility function. Let $x$ denote the interval opinion of DM $i, x \in\left[b_{i 1}, b_{i 3}\right]$, and let $u(x)$ denote the utility of DM $i$ 's opinion, $0 \leq u(x) \leq 1$. Assume that $b_{i 1}, b_{i 2}, b_{i 3}$ are known critical points (reference points or inflection points) within the range of DM $i$ 's opinion. S-shaped utility functions are mainly divided into two types (shown in Fig. 4):

1. left-skewed S-shaped utility function, where the DM's risk attitude shifts from risk-averse to risk-seeking, which means that the marginal utility decreases first and then increases with the increase of DM's opinion whereas the utility of DM is decreasing in general;

2. right-skewed S-shaped utility function, where the DM's risk attitude shifts from risk-seeking to risk-averse, which means that the marginal utility increases first and then decreases with the increase of DM's opinion while the utility of DM is increasing in general.

Expressions of DM's opinion corresponding to these type of S-shaped utility functions are similarly listed in Table 2 .

Table 2: S-shaped utility functions

\begin{tabular}{|c|c|c|}
\hline & Left-skewed S-shaped utility function & Right-skewed S-shaped utility function \\
\hline Utility function & $u(x)=\frac{1}{2}-\frac{1}{2} \sin \frac{\pi}{b_{i 3}-b_{i 1}}\left(x-b_{i 2}\right), b_{i 1} \leq x<b_{i 3}$ & $u(x)=\frac{1}{2}+\frac{1}{2} \sin \frac{\pi}{b_{i 3}-b_{i 1}}\left(x-b_{i 2}\right), b_{i 1} \leq x<b_{i 3}$ \\
\hline DM's opinion & $x=\frac{b_{i 3}-b_{i 1}}{\pi} \arcsin [1-2 u(x)]+b_{i 2}, b_{i 1} \leq x<b_{i 3}$ & $x=\frac{b_{i 3}-b_{i 1}}{\pi} \arcsin [2 u(x)-1]+b_{i 2}, b_{i 1} \leq x<b_{i 3}$ \\
\hline
\end{tabular}




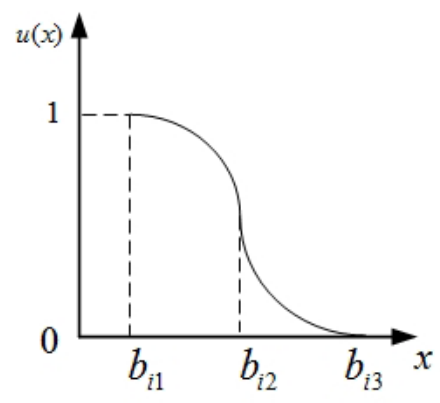

4.1 Left-skewed S-shaped utility function

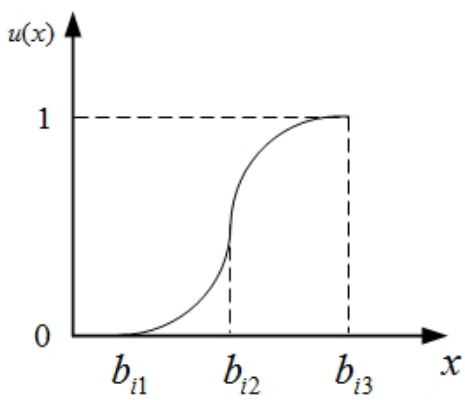

4.2 Right-skewed S-shaped utility function

Fig. 4: Left-skewed and right-skewed S-shaped utility functions

(iii) HARA utility function. Let $x$ denote the opinion of DM $i$, and $u(x)$ is the utility of DM $i$ 's opinion, $0 \leq u(x) \leq 1$. The HARA utility function $[47,55]$ has the following expression

$$
u(x)=\frac{1-\gamma}{\beta \gamma}\left(\frac{\beta}{1-\gamma} x+\eta\right)^{\gamma}, \beta>0, \eta>0, \gamma \in(-\infty, 0) \cup(0,1)
$$

This type of utility function includes as particular cases the power, logarithm, and exponential utility functions by suitable adjustments of its parameters $\beta, \eta, \gamma$ as summarised in Table 3.

Table 3: Three particular types of HARA utility functions with suitable adjustments of the parameters $\beta, \eta, \gamma$

\begin{tabular}{|c|c|c|c|}
\hline & $\eta=0, \beta=1$ & $\eta=0, \beta=1, \gamma \rightarrow 0$ & $\eta=0, \gamma \rightarrow-\infty$ \\
\hline & Power utility function & Logarithm utility function & Exponential utility function \\
\hline Utility function & $u(x)=\frac{x^{\gamma}}{\gamma}, \gamma \in(-\infty, 0) \cup(0,1)$ & $u(x)=\ln (x)$ & $u(x)=e^{-\beta x}, \beta>0$ \\
\hline DM's opinion & $x=\sqrt[\gamma]{\gamma u(x)}$ & $x=e^{u(x)}$ & $x=-\frac{\ln u(x)}{\beta}$ \\
\hline
\end{tabular}

\subsection{Fuzzy Preference Relations: Interval-valued and Intuitionistic}

A preference relation $(\mathrm{PR})$ is based on alternatives pairwise comparisons. The concept of fuzzy preference relation (FPR) to represent intensities of preferences was proposed by Bezdek et al. [56], comprehensively interpreted in Nurmi [57] and broadly studied in [4, 27, 58-60]. These studies rely on the provision of crisp values in $[0,1]$ in constructing FPRs.

Definition 1. A fuzzy preference relation (FPR) $P$ on a finite set of alternatives $X=\left\{x_{1}, \ldots, x_{n}\right\}$ is characterised by a membership function $\mu_{P}: X \times X \longrightarrow[0,1], \mu_{P}\left(x_{i}, x_{j}\right)=p_{i j}$, verifying:

$$
p_{i j}+p_{j i}=1, \forall i, j \in\{1, \ldots, n\} .
$$

The associated semantic for the unit interval of a reciprocal preference relation is assumed to be as follows:

$$
p_{i j}= \begin{cases}0 & \text { if } x_{j} \text { is definitely preferred to } x_{i} \\ p_{i j} \in[0,0.5) & \text { if } x_{j} \text { is preferred to } x_{i} \\ 0.5 & \text { if } x_{i} \text { and } x_{j} \text { are equally preferred (indifference) } \\ p_{i j} \in(0.5,1] & \text { if } x_{i} \text { is preferred to } x_{j} \\ 1 & \text { if } x_{i} \text { is definitely preferred to } x_{j}\end{cases}
$$


A FPR is also known as a reciprocal [0,1]-valued preference relation. However, many decision making problems happen in complex environment pervaded with uncertainty, which can impede the measuring of preference with one single value as required by FPRs. Fortunately, there exists alternative preference representation formats to FPRs more appropriate and flexible to be used in these complex decision making contexts: interval-valued fuzzy preference relations (IVFPRs) and intuitionistic fuzzy preference relations (IFPRs).

\subsubsection{Interval-valued Fuzzy Preference Relation (IVFPR)}

An interval-valued fuzzy preference relation (IVFPR) is defined as follows.

Definition 2. A nonnegative matrix $\bar{R}=\left(\bar{r}_{i j}\right)_{n \times n}=\left(\left[r_{i j}^{L}, r_{i j}^{U}\right]\right)_{n \times n}$ satisfying $\bar{r}_{i i}=[0.5,0.5], r_{i j}^{L}+r_{j i}^{U}=$ $r_{i j}^{U}+r_{j i}^{L}=1, i, j \in N=\{1,2, \ldots, n\}$, is called an interval-valued fuzzy preference relation (IVFPR) .

Judgement elements of an IVFPR $\bar{r}_{i j}=\left[r_{i j}^{L}, r_{i j}^{U}\right]$ denote the extent to which the range of alternative $x_{i}$ is preferred to alternative $x_{j}$, and $r_{i j}^{L}, r_{i j}^{U}$ are lower and upper bounds of $\bar{r}_{i j}$, respectively. Specifically, $\bar{r}_{i j}=[0.5,0.5]$ denotes that there is no difference between alternative $x_{i}$ and alternative $x_{j} ; \bar{r}_{i j}=[1,1]$ denotes that alternatives $x_{i}$ is completely preferred to alternative $x_{j}$; while $\bar{r}_{i j}=[0,0]$ denotes that alternatives $x_{j}$ is completely preferred to alternative $x_{i}$. Besides, if $r_{i j}^{L} \geq 0.5$, alternative $x_{i}$ is preferred to alternative $x_{j}$; and if $r_{i j}^{U} \leq 0.5$, alternative $x_{j}$ is preferred to alternative $x_{i}$.

Given a set of alternatives with associated weight vector (indicating the importance of alternatives), the following result provides the relationship between the concept of multiplicative consistency property of an IVFPR [58] on a set of alternatives and its weight vector. [21]

Proposition 1. Let $\bar{\omega}=\left(\bar{\omega}_{1}, \bar{\omega}_{2}, \ldots, \bar{\omega}_{n}\right)^{T}$ be a weight vector associated to a set alternatives $X=$ $\left\{x_{1}, x_{2}, \ldots, x_{n}\right\}$ such that $\sum_{i=1}^{n} \bar{\omega}_{i}=1, \bar{\omega}_{i}>0, i \in N$. If the IVFPR $\bar{R}$ satisfies multiplicative consistency, the consistency preference $\bar{r}_{i j}(i, j \in N)$ can be obtained as follows

$$
\bar{r}_{i j}=\frac{\bar{\omega}_{i}}{\bar{\omega}_{i}+\bar{\omega}_{j}}, \quad i, j \in N
$$

In Proposition 1, $\bar{r}_{i j}$ as an interval should be transformed into a crisp value.

(1) Without considering the DM's preference. We assume that $\bar{r}_{i j}=\alpha_{i j} r_{i j}^{L}+\left(1-\alpha_{i j}\right) r_{i j}^{U}$, where $0 \leq \alpha_{i j} \leq 1$.

(2) Considering the DM's preference. It is assumed that the DM has a preference for the value of $\bar{r}_{i j}, \bar{r}_{i j}$ in interval $\left[r_{i j}^{L}, r_{i j}^{U}\right]$ represents a utility of DM. Assuming that the utility function $\lambda=u(x)$ in interval $\left[r_{i j}^{L}, r_{i j}^{U}\right]$ is continuously differentiable then $u^{\prime}(x)>0$ and $u^{\prime \prime}(x)<0$. Obviously, each utility value $\lambda$ of DM must correspond to a judgement element $x=u^{-1}(\lambda)$ of $\mathrm{DM}, x \in\left[r_{i j}^{L}, r_{i j}^{U}\right]$. Thus, $\bar{r}_{i j}$ can be written as $\bar{r}_{i j} \doteq u^{-1}(\lambda)$. 


\subsubsection{Intuitionistic fuzzy preference relation (IFPR)}

Intuitionistic fuzzy sets [61] are based on the use of membership degree, non-membership degree and hesitation degree to model experts' subjective preferences, which makes them suitable to model and represent fuzzy and uncertain information.

Definition 3. [61] Given a universe of discourse, $X$, an intuitionistic fuzzy set (IFS) $A$ over $X$

$$
A=\left\{\left\langle x, \mu_{A}(x), \nu_{A}(x)\right\rangle \mid x \in X\right\}
$$

is characterised by a membership function $\mu_{A}: X \rightarrow[0,1]$ and a non-membership function $\nu_{A}: X \rightarrow$ $[0,1]$ verifying:

$$
0 \leq \mu_{A}(x)+\nu_{A}(x) \leq 1 \quad \forall x \in X
$$

When $\mu_{A}(x)=1-\nu_{A}(x) \forall x \in X$, IFSs become fuzzy sets (FSs). If $\exists x \in X: \mu_{A}(x)<1-\nu_{A}(x)$ then the hesitancy function, $\pi_{A}: X \rightarrow[0,1]$, is defined with $\pi_{A}(x)=1-\mu_{A}(x)-\nu_{A}(x)$ interpreted as the hesitation margin of the membership of element $x$ to the IFS $A$, i.e. the amount of lacking information when determining the membership of $x$ to $A$.

Definition 4. [62] An intuitionistic fuzzy preference relation (IFPR) $R$ on a finite set of alternatives $X=\left\{x_{1}, \ldots, x_{n}\right\}$ is characterised by a membership function $\mu_{R}: X \times X \rightarrow[0,1]$ and a non-membership function $\nu_{R}: X \times X \rightarrow[0,1]$ verifying

$$
0 \leq \mu_{R}\left(x_{i}, x_{j}\right)+\nu_{R}\left(x_{i}, x_{j}\right) \leq 1 \quad \forall\left(x_{i}, x_{j}\right) \in X \times X
$$

and (i) $\mu_{i i}=\nu_{i i}=0.5 \forall i \in\{1, \ldots, n\}$; (ii) $\mu_{j i}=\nu_{i j} \forall i, j \in\{1, \ldots, n\}$. The value $\mu_{R}\left(x_{i}, x_{j}\right)=\mu_{i j}$ is interpreted as the certainty degree up to which $x_{i}$ is preferred to $x_{j}$ (membership degree), while the value $\nu_{R}\left(x_{i}, x_{j}\right)=\nu_{i j}$ represents the certainty degree up to which $x_{i}$ is non-preferred to $x_{j}$ (nonmembership degree). Using matrix notation, an IFPR is represented as $R=\left(r_{i j}\right)=\left(\left\langle\mu_{i j}, \nu_{i j}\right\rangle\right)$.

An IFPR is usually represented in matrix form

$$
R=\left(\mu_{i j}, \nu_{i j}, \pi_{i j}\right)_{n \times n}=\left(\begin{array}{cccc}
\left(\mu_{11}, \nu_{11}, \pi_{11}\right) & \left(\mu_{12}, \nu_{12}, \pi_{12}\right) & \ldots & \left(\mu_{1 n}, \nu_{1 n}, \pi_{1 n}\right) \\
\left(\mu_{21}, \nu_{21}, \pi_{21}\right) & \left(\mu_{22}, \nu_{22}, \pi_{22}\right) & \ldots & \left(\mu_{2 n}, \nu_{2 n}, \pi_{2 n}\right) \\
\vdots & \vdots & & \vdots \\
\left(\mu_{n 1}, \nu_{n 1}, \pi_{n 1}\right) & \left(\mu_{n 2}, \nu_{n 2}, \pi_{n 2}\right) & \ldots & \left(\mu_{n n}, \nu_{n n}, \pi_{n n}\right)
\end{array}\right)
$$

The hesitancy index is a 'regulator', by which the DM can increase or decrease their judgement. This is equivalent to stating that the membership degree and non-membership degree lie in the closed intervals $\left[\mu_{i j}, \mu_{i j}+\pi_{i j}\right]$ and $\left[\nu_{i j}, \nu_{i j}+\pi_{i j}\right]$. Consequently, IFPR $R$ can be equivalently transformed into the membership IVFPR $A$ and the non-membership IVFPR $B$ :

$$
A=\left(a_{i j}\right)_{n \times n}=\left(\left[a_{i j}^{L}, a_{i j}^{U}\right]\right)_{n \times n}
$$




$$
B=\left(b_{i j}\right)_{n \times n}=\left[b_{i j}^{L}, b_{i j}^{U}\right]_{n \times n}
$$

where $a_{i j}^{L}=\mu_{i j}, a_{i j}^{U}=\mu_{i j}+\pi_{i j}, b_{i j}^{L}=\nu_{i j}$, and $b_{i j}^{U}=\nu_{i j}+\pi_{i j}$. Indeed, it is

$$
\begin{aligned}
& {\left[\mu_{i i}, \mu_{i i}+\pi_{i i}\right]=[0.5,0.5], \mu_{i j}+\left(\mu_{j i}+\pi_{j i}\right)=\left(\mu_{i j}+\pi_{i j}\right)+\mu_{j i}=1, \quad i, j \in N} \\
& {\left[\nu_{i i}, \nu_{i i}+\pi_{i i}\right]=[0.5,0.5], \quad \nu_{i j}+\left(\nu_{j i}+\pi_{j i}\right)=\left(\nu_{i j}+\pi_{i j}\right)+\nu_{j i}=1, \quad i, j \in N}
\end{aligned}
$$

which proves that both $A$ and $B$ are IVFPRs. Thus, IVFPRs $A$ and $B$ can be used to replace an IFPR $R$ equivalently.

Lemma 1. Given an IFPR $R$, IVFPRs $A=\left[a_{i j}^{L}, a_{i j}^{U}\right]$ and $B=\left[b_{i j}^{L}, b_{i j}^{U}\right]$ are equivalent to $R$ iff

$$
a_{i j}^{L}+b_{i j}^{U}=1, \quad b_{i j}^{L}+a_{i j}^{U}=1, \quad i, j \in N
$$

\subsection{Relationship between IFPRs and utility functions}

In consideration of DM's risk attitude and psychological factors, this paper adopts the DM's utility function taking value in intuitionistic fuzzy numbers, and achieves the goal of selecting and classifying alternatives. This section explores relevant results about IFPRs based on utility functions. Similarly, IFPR is transformed into equivalent membership and non-membership IVFPRs. As a particular utility function reflects a particular DM's subjective risk factor, the preference information of the membership IVFPR corresponds to a utility function whereas the non-membership IVFPR corresponds to another utility function.

Theorem 1. Assume that the equivalent membership IVFRP $A=\left(a_{i j}\right)_{n \times n}$ and non-membership IVFPR $B=\left(b_{i j}\right)_{n \times n}$ of the IFPR R satisfy utility function $\lambda^{A}=u\left(a_{i j}\right)$ and $\lambda^{B}=u\left(b_{i j}\right)$, respectively. Then $a_{i j}=u^{-1}\left(\lambda^{A}\right), b_{i j}=u^{-1}\left(\lambda^{B}\right)$, we have $u^{-1}\left(\lambda^{A}\right)+u^{-1}\left(\lambda^{B}\right)=1$.

Proof. Let $r_{i j}=\left(\mu_{i j}, \nu_{i j}, \pi_{i j}\right)$ be an intuitionistic fuzzy element in $R$; we have the equivalent membership degree interval $a_{i j}=\left[a_{i j}^{L}, a_{i j}^{U}\right]$ and non-membership degree interval $b_{i j}=\left[b_{i j}^{L}, b_{i j}^{U}\right]$. Because

$$
b_{i j}=u^{-1}\left(\lambda^{B}\right)=\left[b_{i j}^{L}, b_{i j}^{U}\right]=1-\left[a_{i j}^{L}, a_{i j}^{U}\right]=1-a_{i j}=1-u^{-1}\left(\lambda^{A}\right)
$$

thus $u^{-1}\left(\lambda^{A}\right)+u^{-1}\left(\lambda^{B}\right)=1$ holds.

Theorem 2. Assume that the equivalent membership IVFPR $A=\left(a_{i j}\right)_{n \times n}$ and non-membership IVFPR $B=\left(b_{i j}\right)_{n \times n}$ of the IFPR $R$ satisfy utility function $\lambda^{A}=u\left(a_{i j}\right)$ and $\lambda^{B}=u\left(b_{i j}\right)$, respectively. If corresponding weight vectors $\omega^{A}=\left(\omega_{1}^{A}, \ldots, \omega_{n}^{A}\right)^{T}\left(\sum_{i=1}^{n} \omega_{i}^{A}=1,0 \leq \omega_{i}^{A} \leq 1\right)$ and $\omega^{B}=$ $\left(\omega_{1}^{B}, \ldots, \omega_{n}^{B}\right)^{T}\left(\sum_{i=1}^{n} \omega_{i}^{B}=1,0 \leq \omega_{i}^{B} \leq 1\right)$ satisfy

$$
\begin{aligned}
& \frac{\omega_{i}^{A}}{\omega_{i}^{A}+\omega_{j}^{A}}=u^{-1}\left(\lambda^{A}\right), \quad i, j \in N ; \\
& \frac{\omega_{i}^{B}}{\omega_{i}^{B}+\omega_{j}^{B}}=u^{-1}\left(\lambda^{B}\right), \quad i, j \in N^{\prime}
\end{aligned}
$$

then the IFPR $R$ satisfies multiplicative consistency. 
Proof. From $\lambda^{A}=u\left(a_{i j}\right)$ and $\lambda^{B}=u\left(b_{i j}\right)$, it is $a_{i j}=u^{-1}\left(\lambda^{A}\right)$ and $b_{i j}=u^{-1}\left(\lambda^{B}\right)$. In addition, we have $a_{i j}=\frac{\omega_{i}^{A}}{\omega_{i}^{A}+\omega_{j}^{A}}$ and $b_{i j}=\frac{\omega_{i}^{B}}{\omega_{i}^{B}+\omega_{j}^{B}}$. As per Proposition 1, IVFPRs $A=\left(a_{i j}\right)_{n \times n}$ and $B=$ $\left(b_{i j}\right)_{n \times n}$ satisfy multiplicative consistency. Thus, the corresponding IFPR $R$ also satisfes multiplicative consistency.

\section{Optimization ordering model for IFPRs based on non-linear utility functions}

To ensure the reliability and accuracy of the ranking of IFPRs, the consistency of IFPRs must be considered; non-linear utility functions can better represent the preference utility of different DMs. Therefore, it is more effective to obtain the ranking of alternatives that reflect DMs' preferences by combining consistency and utility preference. In this section, $a_{i j}$ denotes the actual judgment value of IFPRs given by a DM, satisfying non-linear utility function $u(x)$; and $a_{i j}^{*}=\frac{\omega_{i}}{\omega_{i}+\omega_{j}}$ designates the ideal judgment value obeying multiplicative consistency. Under the premise of satisfying DMs' different utilities, to minimize the distance deviation between the ideal judgment value obeying multiplicative consistency and the actual judgment value of IFPRs, the optimization ordering model for IFPRs is constructed in the case of non-consistency.

\subsection{Steps of the optimization ordering modelling for IFPRs based on non-linear utility functions}

Step 1: In GDM, the IFPRs given by the DM $k$ are $R^{k}=\left(r_{i j}^{k}\right)_{n \times n}=\left(\mu_{i j}^{k}, \nu_{i j}^{k}, \pi_{i j}^{k}\right)_{n \times n}$.

Step 2: Construct the equivalent IVFPRs to $R^{k}$, i.e., the membership IVFPR $A^{k}=\left(a_{i j}^{k}\right)_{n \times n}=$ $\left[\mu_{i j}^{k}, \mu_{i j}^{k}+\pi_{i j}^{k}\right]_{n \times n}$ and the non-membership IVFPR $B^{k}=\left(b_{i j}^{k}\right)_{n \times n}=\left[\nu_{i j}^{k}, \nu_{i j}^{k}+\pi_{i j}^{k}\right]_{n \times n}$.

Step 3: Construct utility functions $\lambda_{A}^{k}=u\left(a_{i j}^{k}\right)_{n \times n}$ and $\lambda_{B}^{k}=u\left(b_{i j}^{k}\right)_{n \times n}$ of the membership and non-membership IVFPRs $A^{k}$ and $B^{k}$, respectively, using parabolic, S-shaped and HARA utility function.

Step 4: Assuming $\omega^{A}=\left(\omega_{1}^{A}, \omega_{2}^{A}, \ldots, \omega_{n}^{A}\right)^{T}$ and $\omega^{B}=\left(\omega_{1}^{B}, \omega_{2}^{B}, \ldots, \omega_{n}^{B}\right)^{T}$ are the ranking weight vectors of $A^{k}$ and $B^{k}$, respectively, to minimise the distance deviation between the ideal judgment value obeying multiplicative consistency and the actual judgment value of IFPRs, the optimization ordering models for IFPRs based on nonlinear utility functions are built as follows:

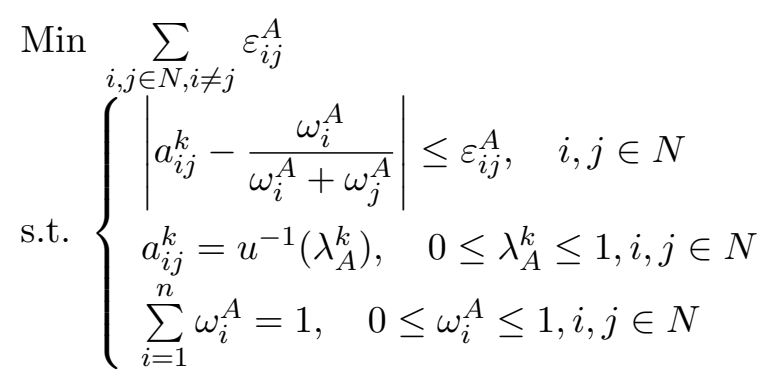




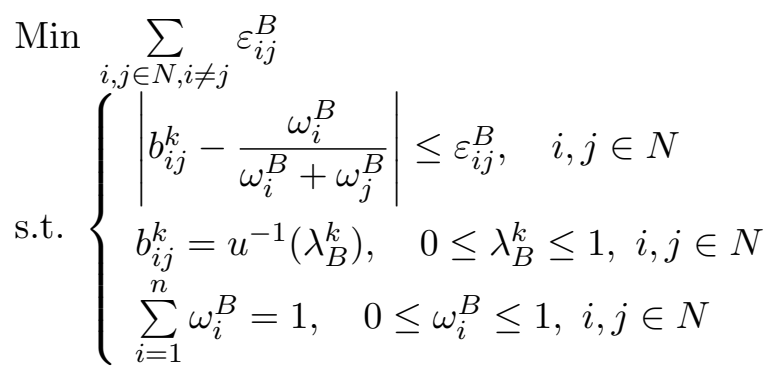

Model (1) and (2) are the optimization ordering models for the membership and non-membership IVFPRs based on nonlinear utility functions, jointly devoting to the optimal ranking for IFPR. In Model (1), Constraint (1-1) indicates that the distance deviation between the ideal judgment value obeying multiplicative consistency $\frac{\omega_{i}^{A}}{\omega_{i}^{A}+\omega_{J}^{A}}$ and the actual judgment value $a_{i j}^{k}$ of the membership IVFPR $A^{k}$ is limited in a threshold $\varepsilon_{i j}^{A}$. Constraint (1-2) means that the actual judgment value $a_{i j}^{k}$ satisfies the utility function $u^{-1}\left(\lambda_{A}^{k}\right)$, and Constraint (1-3) put restrictions on the ranking weights. Consequently, the objective function is the sum of thresholds $\sum_{i, j \in N, i \neq j} \varepsilon_{i j}^{A}$ for all elements in the membership IVFPR, which is to be minimized, corresponding to a more accurate ranking. And the interpretation for Model (2) follows from Model (1).

\subsection{Optimization ordering model for IFPRs based on various non-linear utility functions}

\subsubsection{Optimization ordering model for IFPRs based on a parabolic utility function}

Taking the right-skewed parabolic utility function as per Table 1, the optimization ordering model for the membership IVFPR $A^{k}$ and the non-membership IVFPR $B^{k}$ become:

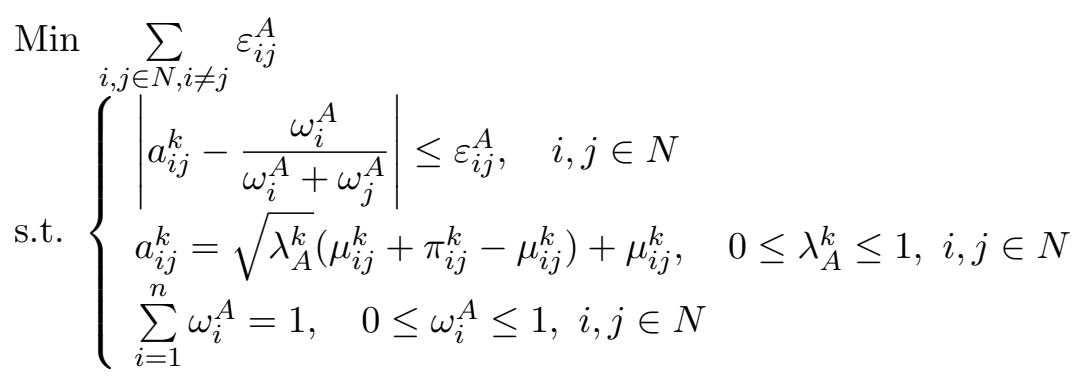

$$
\begin{array}{ll}
\text { Min } & \sum_{i, j \in N, i \neq j} \varepsilon_{i j}^{B} \\
\text { s.t. }\left\{\begin{array}{l}
\left|b_{i j}^{k}-\frac{\omega_{i}^{B}}{\omega_{i}^{B}+\omega_{j}^{B}}\right| \leq \varepsilon_{i j}^{B}, \quad i, j \in N \\
b_{i j}^{k}=\sqrt{\lambda_{B}^{k}}\left(\nu_{i j}^{k}+\pi_{i j}^{k}-\nu_{i j}^{k}\right)+\nu_{i j}^{k}, \quad 0 \leq \lambda_{B}^{k} \leq 1, i, j \in N \\
\sum_{i=1}^{n} \omega_{i}^{B}=1, \quad 0 \leq \omega_{i}^{B} \leq 1, i, j \in N
\end{array}\right.
\end{array}
$$




\subsubsection{Optimization ordering model for IFPRs based on an S-shaped utility function}

Taking the right-skewed S-shaped utility function as per Table 2, the optimization ordering model for the membership IVFPR $A^{k}$ and the non-membership IVFPR $B^{k}$ become:

$$
\begin{array}{ll}
\operatorname{Min} & \sum_{i, j \in N, i \neq j} \varepsilon_{i j}^{A} \\
\text { s.t. } & \left\{\begin{array}{l}
\left|a_{i j}^{k}-\frac{\omega_{i}^{A}}{\omega_{i}^{A}+\omega_{j}^{A}}\right| \leq \varepsilon_{i j}^{A}, \quad i, j \in N \\
a_{i j}^{k}=\frac{\mu_{i j}^{k}+\pi_{i j}^{k}-\mu_{i j}^{k}}{\pi} \arcsin \left(2 \lambda_{A}^{k}-1\right)+\frac{\mu_{i j}^{k}+\pi_{i j}^{k}+\mu_{i j}^{k}}{2}, \quad 0 \leq \lambda_{A}^{k} \leq 1, i, j \in N \\
\sum_{i=1}^{n} \omega_{i}^{A}=1, \quad 0 \leq \omega_{i}^{A} \leq 1, i, j \in N
\end{array}\right.
\end{array}
$$

$$
\begin{array}{ll}
\operatorname{Min} & \sum_{i, j \in N, i \neq j} \varepsilon_{i j}^{B} \\
\text { s.t. }\left\{\begin{array}{l}
\left|b_{i j}^{k}-\frac{\omega_{i}^{B}}{\omega_{i}^{B}+\omega_{j}^{B}}\right| \leq \varepsilon_{i j}^{B}, \quad i, j \in N \\
b_{i j}^{k}=\frac{\nu_{i j}^{k}+\pi_{i j}^{k}-\nu_{i j}^{k}}{\pi} \arcsin \left(2 \lambda_{B}^{k}-1\right)+\frac{\nu_{i j}^{k}+\pi_{i j}^{k}+\nu_{i j}^{k}}{2}, \quad 0 \leq \lambda_{B}^{k} \leq 1, i, j \in N \\
\sum_{i=1}^{n} \omega_{i}^{B}=1, \quad 0 \leq \omega_{i}^{B} \leq 1, i, j \in N
\end{array}\right.
\end{array}
$$

3.3. Relationship between the optimization ordering model for membership and non-membership IVF-

PRs based on non-linear utility functions

Model (3) and (4) can be expressed as follows:

$$
\begin{aligned}
& \text { Min } \quad \sum_{i, j \in N, i \neq j} \varepsilon_{i j}^{A} \\
& \text { s.t. }\left\{\begin{array}{l}
\left|\sqrt{\lambda_{A}^{k}}\left(\mu_{i j}^{k}+\pi_{i j}^{k}-\mu_{i j}^{k}\right)+\mu_{i j}^{k}-\frac{\omega_{i}^{A}}{\omega_{i}^{A}+\omega_{j}^{A}}\right| \leq \varepsilon_{i j}^{A}, \quad i, j \in N \\
\sum_{i=1}^{n} \omega_{i}^{A}=1, \quad 0 \leq \omega_{i}^{A} \leq 1,0 \leq \lambda_{A}^{k} \leq 1, \quad i, j \in N
\end{array}\right. \\
& \text { Min } \quad\left\{\begin{array}{l}
\sum_{i, j \in N, i \neq j} \varepsilon_{i j}^{B} \\
\text { s.t. } \sqrt{\lambda_{B}^{k}}\left(\nu_{i j}^{k}+\pi_{i j}^{k}-\nu_{i j}^{k}\right)+\nu_{i j}^{k}-\frac{\omega_{i}^{B}}{\omega_{i}^{B}+\omega_{j}^{B}} \mid \leq \varepsilon_{i j}^{B}, \quad i, j \in N \\
\sum_{i=1}^{n} \omega_{i}^{B}=1, \quad 0 \leq \omega_{i}^{B} \leq 1,0 \leq \lambda_{B}^{k} \leq 1, i, j \in N
\end{array}\right.
\end{aligned}
$$

Because

$$
\sqrt{\lambda_{B}^{k}}\left(\nu_{i j}^{k}+\pi_{i j}^{k}-\nu_{i j}^{k}\right)+\nu_{i j}^{k}=b_{i j}=\left[b_{i j}^{L}, b_{i j}^{U}\right]=1-\left[a_{i j}^{L}, a_{i j}^{U}\right]=1-a_{i j}=1-\sqrt{\lambda_{A}^{k}}\left(\mu_{i j}^{k}+\pi_{i j}^{k}-\mu_{i j}^{k}\right)+\mu_{i j}^{k}
$$

We have

$$
\begin{aligned}
\left|\frac{\omega_{i}^{B}}{\omega_{i}^{B}+\omega_{j}^{B}}-\left[\sqrt{\lambda_{B}^{k}}\left(\nu_{i j}^{k}+\pi_{i j}^{k}-\nu_{i j}^{k}\right)+\nu_{i j}^{k}\right]\right| & =\left|1-\frac{\omega_{j}^{B}}{\omega_{i}^{B}+\omega_{j}^{B}}-\left\{1-\left[\sqrt{\lambda_{B}^{k}}\left(\nu_{i j}^{k}+\pi_{i j}^{k}-\nu_{i j}^{k}\right)+\nu_{i j}^{k}\right]\right\}\right| \\
& =\left|-\frac{\omega_{j}^{B}}{\omega_{i}^{B}+\omega_{j}^{B}}+\left[\sqrt{\lambda_{A}^{k}}\left(\mu_{i j}^{k}+\pi_{i j}^{k}-\mu_{i j}^{k}\right)+\mu_{i j}^{k}\right]\right| \\
& =\left|\frac{\omega_{j}^{B}}{\omega_{i}^{B}+\omega_{j}^{B}}-\left[\sqrt{\lambda_{A}^{k}}\left(\mu_{i j}^{k}+\pi_{i j}^{k}-\mu_{i j}^{k}\right)+\mu_{i j}^{k}\right]\right|
\end{aligned}
$$


Let $\frac{\omega_{i}^{B}}{\omega_{i}^{B}+\omega_{j}^{B}}=x_{i j}$ and $\frac{\omega_{j}^{B}}{\omega_{i}^{B}+\omega_{j}^{B}}=y_{j i}$, then the expression (9) coincides with expression (7-1). The following theorems can be obtained.

Theorem 3. The solutions and optimal values of multiplicative consistency for ordering models for the equivalent membership IVFPR A and non-membership IVFPR B of the IFPR $R$ based on non-linear utility functions coincide.

Proof. As per Theorem 1, because

$$
b_{i j}=u^{-1}\left(\lambda^{B}\right)=\left[b_{i j}^{L}, b_{i j}^{U}\right]=1-\left[a_{i j}^{L}, a_{i j}^{U}\right]=1-a_{i j}=1-u^{-1}\left(\lambda^{A}\right)
$$

it is

$$
\begin{aligned}
\left|\frac{\omega_{i}^{B}}{\omega_{i}^{B}+\omega_{j}^{B}}-u^{-1}\left(\lambda^{B}\right)\right| & =\left|1-\frac{\omega_{j}^{B}}{\omega_{i}^{B}+\omega_{j}^{B}}-\left[1-u^{-1}\left(\lambda^{A}\right)\right]\right|=\left|-\frac{\omega_{j}^{B}}{\omega_{i}^{B}+\omega_{j}^{B}}+u^{-1}\left(\lambda^{A}\right)\right| \\
& =\left|\frac{\omega_{j}^{B}}{\omega_{i}^{B}+\omega_{j}^{B}}-u^{-1}\left(\lambda^{A}\right)\right|
\end{aligned}
$$

Let $\frac{\omega_{i}^{A}}{\omega_{i}^{A}+\omega_{j}^{A}}=x_{i j}$ and $\frac{\omega_{j}^{B}}{\omega_{i}^{B}+\omega_{j}^{B}}=y_{j i}$, thenexpression (10) coincides with expression (1-1).

Theorem 4. The ranking solutions of the equivalent membership IVFPR A and non-membership IVFPR $B$ of the IFPR $R$ with multiplicative consistency derived by optimization ordering models based on non-linear utility function have a reversal relationship.

Proof. From Theorem 3, $\frac{\omega_{i}^{A}}{\omega_{i}^{A}+\omega_{j}^{A}}=\frac{\omega_{j}^{B}}{\omega_{i}^{B}+\omega_{j}^{B}}$, namely, $\frac{\omega_{i}^{A}}{\omega_{j}^{A}}=\frac{\omega_{j}^{B}}{\omega_{i}^{B}}$. Hence, in Model (7), if there exists a weight vector $\omega_{\sigma 1}^{A} \geq \omega_{\sigma 2}^{A} \geq \cdots \geq \omega_{\sigma i}^{A} \geq \cdots \geq \omega_{\sigma n}^{A}, i \in N$, then the weight vector of Model (8) satisfies $\omega_{\sigma 1}^{B} \leq \omega_{\sigma 2}^{B} \leq \cdots \leq \omega_{\sigma i}^{B} \leq \cdots \leq \omega_{\sigma n}^{B}, i \in N$.

\section{Optimization ordering model for IFPRs under the priority of utility and distance deviation}

As the DM's fuzzy judgement satisfies a utility function, achieving utility maximisation is one of the goals expected by every DM. Therefore, based on the realisation of the single objective (minimising the distance deviation between the ideal judgment value obeying multiplicative consistency and the actual judgment value of IFPRs), this section further investigates the optimization ordering modeling for IFPRs with both targets of utility maximisation and distance deviation minimisation.

In the extended real number field, non-Archimedean infinitesimal $\tau$ is a number, which is smaller than any positive number and greater than zero. By introducing non-Archimedean infinitesimal $\tau$ [63] to the optimization ordering model for IFPRs based on non-linear utility functions, the optimization ordering models for IFPRs under the priority of utility and distance deviation are structured, respectively. 


\subsection{Optimization ordering model for IFPRs under the priority of utility}

Based on Models (1) and (2), we give priority to the goal of utility maximisation, i.e. introducing non-Archimedean infinitesimal $\tau$ to the distance deviation $\varepsilon_{i j}$, and realise the optimal ranking for IFPRs under the priority of utility:

$$
\begin{aligned}
& \text { Min }-\lambda_{A}^{k}+\sum_{i, j \in N, i \neq j} \tau \varepsilon_{i j}^{A} \\
& \text { s.t. }\left\{\begin{array}{l}
\left|a_{i j}^{k}-\frac{\omega_{i}^{A}}{\omega_{i}^{A}+\omega_{j}^{A}}\right| \leq \varepsilon_{i j}^{A}, \quad i, j \in N \\
a_{i j}^{k}=u^{-1}\left(\lambda_{A}^{k}\right), \quad 0 \leq \lambda_{A}^{k} \leq 1, i, j \in N \\
\sum_{i=1}^{n} \omega_{i}^{A}=1, \quad 0 \leq \omega_{i}^{A} \leq 1, i, j \in N
\end{array}\right. \\
& \text { Min }-\lambda_{B}^{k}+\sum_{i, j \in N, i \neq j} \tau \varepsilon_{i j}^{B} \\
& \text { s.t. }\left\{\begin{array}{l}
\left|b_{i j}^{k}-\frac{\omega_{i}^{B}}{\omega_{i}^{B}+\omega_{j}^{B}}\right| \leq \varepsilon_{i j}^{B}, i, j \in N \\
b_{i j}^{k}=u^{-1}\left(\lambda_{B}^{k}\right), \quad 0 \leq \lambda_{B}^{k} \leq 1, i, j \in N \\
\sum_{i=1}^{n} \omega_{i}^{B}=1 \quad, 0 \leq \omega_{i}^{B} \leq 1, i, j \in N
\end{array}\right.
\end{aligned}
$$

In Model (11), the objective function can be interpreted that realizing the maximization of utility under the influence of distance deviation. And the interpretation for Model (12) follows from Model $(11)$.

\subsection{Optimization ordering model for IFPRs under the priority of deviation}

Similarly, giving priority to the goal of distance deviation minimisation, i.e. introducing nonArchimedean infinitesimal $\tau$ to utility $\lambda^{k}$, and the optimal ranking for IFPRs under the priority of distance deviation is obtained:

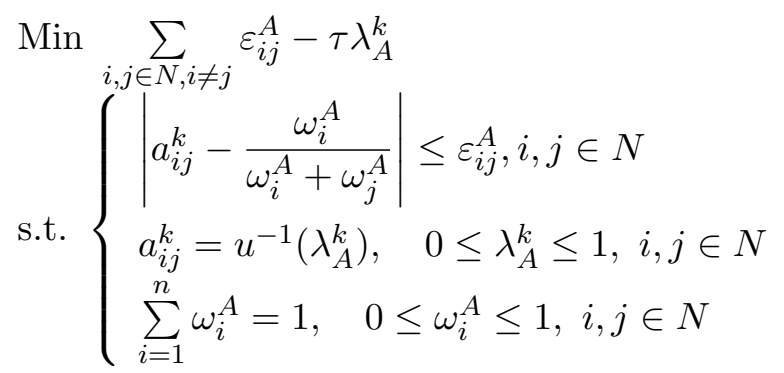

$$
\begin{aligned}
& \operatorname{Min} \sum_{i, j \in N, i \neq j} \varepsilon_{i j}^{B}-\tau \lambda_{B}^{k} \\
& \text { s.t. }\left\{\begin{array}{l}
\left|b_{i j}^{k}-\frac{\omega_{i}^{B}}{\omega_{i}^{B}+\omega_{j}^{B}}\right| \leq \varepsilon_{i j}^{B}, i, j \in N \\
b_{i j}^{k}=u^{-1}\left(\lambda_{B}^{k}\right), \quad 0 \leq \lambda_{B}^{k} \leq 1, i, j \in N \\
\sum_{i=1}^{n} \omega_{i}^{B}=1, \quad 0 \leq \omega_{i}^{B} \leq 1, i, j \in N
\end{array}\right.
\end{aligned}
$$


In Model (13), the objective function can be interpreted that realizing the minimization of distance deviation under the influence of utility. And the interpretation for Model (14) follows from Model $(13)$.

Corollary 1. Assume the equivalent membership IVFPR $A=\left(a_{i j}\right)_{n \times n}$ and non-membership IVFPR $B=\left(b_{i j}\right)_{n \times n}$ to the IFPR $R$ satisfy one of the following utility functions:

- right-skewed parabolic utility function;

- right-skewed S-shaped utility function;

- power utility function;

- logarithm utility function;

- exponential utility function;

then the optimal solutions to the optimization ordering model for IFPRs under the priority of deviation and the optimization ordering model for IFPRs are the same.

\section{Application to global suppliers selection}

In a globalised market environment, global suppliers take various measures to enhance their competitiveness for the sake of maintaining long-term business relations. Measures such as decreasing total cost of supply chain, reducing enterprise inventory, strengthening enterprise information sharing, and improving enterprise interaction are implemented. Therefore, it is of vital importance to establish the optimal evaluation index for global supplier selection [64]. However, global supplier selection is a complex multi-criteria decision problem, relating to different fuzzy expressions. In this case, the manufacturer requires a expert to evaluate candidate global suppliers.

Supposed that the expert builds IFPRs for four candidate suppliers $s_{1}, s_{2}, s_{3}, s_{4}$ with regards to three criteria (such as product's cost, product's quality and supplier's service), as listed below[65].

$$
\begin{aligned}
R^{(1)}= & \left(\begin{array}{cccc}
(0.5,0.5,0) & (0.5,0.2,0.3) & (0.7,0.1,0.2) & (0.5,0.3,0.2) \\
(0.2,0.5,0.3) & (0.5,0.5,0) & (0.6,0.2,0.2) & (0.3,0.6,0.1) \\
(0.1,0.7,0.2) & (0.2,0.6,0.2) & (0.5,0.5,0) & (0.3,0.6,0.1) \\
(0.3,0.5,0.2) & (0.6,0.3,0.1) & (0.6,0.3,0.1) & (0.5,0.5,0)
\end{array}\right) \\
R^{(2)}= & \left(\begin{array}{cccc}
(0.5,0.5,0) & (0.6,0.1,0.3) & (0.8,0.2,0) & (0.6,0.3,0.1) \\
(0.1,0.6,0.3) & (0.5,0.5,0) & (0.5,0.1,0.4) & (0.3,0.7,0) \\
(0.2,0.8,0) & (0.1,0.5,0.4) & (0.5,0.5,0) & (0.4,0.6,0) \\
(0.3,0.6,0.1) & (0.7,0.3,0) & (0.6,0.4,0) & (0.5,0.5,0)
\end{array}\right)
\end{aligned}
$$




$$
R^{(3)}=\left(\begin{array}{cccc}
(0.5,0.5,0) & (0.6,0.2,0.2) & (0.8,0.1,0.1) & (0.7,0.2,0.1) \\
(0.2,0.6,0.2) & (0.5,0.5,0) & (0.6,0.1,0.3) & (0.2,0.7,0.1) \\
(0.1,0.8,0.1) & (0.1,0.6,0.3) & (0.5,0.5,0) & (0.2,0.3,0.5) \\
(0.2,0.7,0.1) & (0.7,0.2,0.1) & (0.3,0.2,0.5) & (0.5,0.5,0)
\end{array}\right)
$$

\subsection{Optimization ordering model for IFPRs based on non-linear utility functions}

For the convenience of calculation and comparison, we assume that all experts utility functions are right-skewed parabolic type. For the purpose of minimizing the distance deviation between the ideal judgment value obeying multiplicative consistency and the actual judgment value by experts, the paper constructs models as per the four steps in Section 4.1, Models (3) and (4). The optimal solutions for the expert's IFPRs $R^{(1)}, R^{(2)}, R^{(3)}$ with regards to three criteria are listed in Table 4.

Table 4: Optimal solutions to ordering models for the membership and non-membership IVFPR for $R^{(1)}, R^{(2)}$, and $R^{(3)}$

\begin{tabular}{|c|c|c|c|c|c|c|c|c|c|c|c|c|}
\hline \multirow{4}{*}{$R^{(1)}$} & $\omega_{1}^{A}$ & $\omega_{2}^{A}$ & $\omega_{3}^{A}$ & $\omega_{4}^{A}$ & $\varepsilon_{12}^{A}$ & $\varepsilon_{13}^{A}$ & $\varepsilon_{14}^{A}$ & $\varepsilon_{23}^{A}$ & $\varepsilon_{24}^{A}$ & $\varepsilon_{34}^{A}$ & $\lambda_{A}^{(1)}$ & $W_{A}^{(1)}$ \\
\hline & 0.3873 & 0.2308 & 0.1065 & 0.2754 & 0 & 0 & 0 & 0 & 0.1138 & 0.0634 & 0.1779 & 0.1772 \\
\hline & $\omega_{1}^{B}$ & $\omega_{2}^{B}$ & $\omega_{3}^{B}$ & $\omega_{4}^{B}$ & $\varepsilon_{12}^{B}$ & $\varepsilon_{13}^{B}$ & $\varepsilon_{14}^{B}$ & $\varepsilon_{23}^{B}$ & $\varepsilon_{24}^{B}$ & $\varepsilon_{34}^{B}$ & $\lambda_{B}^{(1)}$ & $W_{B}^{(1)}$ \\
\hline & 0.1295 & 0.2173 & 0.4711 & 0.1821 & 0 & 0 & 0 & 0 & 0.1138 & 0.0634 & 0.3343 & 0.1772 \\
\hline \multirow{4}{*}{$R^{(2)}$} & $\omega_{1}^{A}$ & $\omega_{2}^{A}$ & $\omega_{3}^{A}$ & $\omega_{4}^{A}$ & $\varepsilon_{12}^{A}$ & $\varepsilon_{13}^{A}$ & $\varepsilon_{14}^{A}$ & $\varepsilon_{23}^{A}$ & $\varepsilon_{24}^{A}$ & $\varepsilon_{34}^{A}$ & $\lambda_{A}^{(2)}$ & $W_{A}^{(2)}$ \\
\hline & 0.4426 & 0.1903 & 0.1106 & 0.2565 & 0 & 0 & 0 & 0 & 0.1260 & 0.0986 & 0.1095 & 0.2246 \\
\hline & $\omega_{1}^{B}$ & $\omega_{2}^{B}$ & $\omega_{3}^{B}$ & $\omega_{4}^{B}$ & $\varepsilon_{12}^{B}$ & $\varepsilon_{13}^{B}$ & $\varepsilon_{14}^{B}$ & $\varepsilon_{23}^{B}$ & $\varepsilon_{24}^{B}$ & $\varepsilon_{34}^{B}$ & $\lambda_{B}^{(2)}$ & $W_{B}^{(2)}$ \\
\hline & 0.1105 & 0.2569 & 0.4420 & 0.1906 & 0 & 0 & 0 & 0 & 0.1260 & 0.0986 & 0.4476 & 0.2246 \\
\hline \multirow{4}{*}{$R^{(3)}$} & $\omega_{1}^{A}$ & $\omega_{2}^{A}$ & $\omega_{3}^{A}$ & $\omega_{4}^{A}$ & $\varepsilon_{12}^{A}$ & $\varepsilon_{13}^{A}$ & $\varepsilon_{14}^{A}$ & $\varepsilon_{23}^{A}$ & $\varepsilon_{24}^{A}$ & $\varepsilon_{34}^{A}$ & $\lambda_{A}^{(3)}$ & $W_{A}^{(3)}$ \\
\hline & 0.4925 & 0.2264 & 0.1000 & 0.1811 & 0.0228 & 0 & 0 & 0 & 0.3244 & 0 & 0.0972 & 0.3472 \\
\hline & $\omega_{1}^{B}$ & $\omega_{2}^{B}$ & $\omega_{3}^{B}$ & $\omega_{4}^{B}$ & $\varepsilon_{12}^{B}$ & $\varepsilon_{13}^{B}$ & $\varepsilon_{14}^{B}$ & $\varepsilon_{23}^{B}$ & $\varepsilon_{24}^{B}$ & $\varepsilon_{34}^{B}$ & $\lambda_{B}^{(3)}$ & $W_{B}^{(3)}$ \\
\hline & 0.0924 & 0.2011 & 0.4551 & 0.2514 & 0.0228 & 0 & 0 & 0 & 0.3244 & 0 & 0.4737 & 0.3472 \\
\hline
\end{tabular}

We notice the following:

1. Correctness of Theorem 1 is verified because $\sqrt{\lambda_{A}^{(1) *}}+\sqrt{\lambda_{B}^{(1) *}}=1 ; \sqrt{\lambda_{A}^{(2) *}}+\sqrt{\lambda_{B}^{(2) *}}=1$; and $\sqrt{\lambda_{A}^{(3) *}}+\sqrt{\lambda_{B}^{(3) *}}=1$

2. Theorem 3 correctness is verified because: $W_{A}^{(1) *}=W_{B}^{(1) *} ; W_{A}^{(2) *}=W_{B}^{(2) *} ;$ and $W_{A}^{(3) *}=W_{B}^{(3) *}$.

3. Theorem 4 correctness is verified because $\omega_{1}^{A *}>\omega_{4}^{A *}>\omega_{2}^{A *}>\omega_{3}^{A *}, \omega_{3}^{B *}>\omega_{2}^{B *}>\omega_{4}^{B *}>\omega_{1}^{B *}$; $\omega_{1}^{A *}>\omega_{4}^{A *}>\omega_{2}^{A *}>\omega_{3}^{A *}, \omega_{3}^{B *}>\omega_{2}^{B *}>\omega_{4}^{B *}>\omega_{1}^{B *}$ in $R^{(2)} ;$ and $\omega_{1}^{A *}>\omega_{2}^{A *}>\omega_{4}^{A *}>\omega_{3}^{A *}$, $\omega_{3}^{B *}>\omega_{4}^{B *}>\omega_{2}^{B *}>\omega_{1}^{B *}$ in $R^{(3)}$.

Moreover, through a comprehensive analysis of Table 4, it is stated that the ranking about criterion 1 is $s_{1} \succ s_{4} \succ s_{2} \succ s_{3}$, the ranking about criterion 2 is $s_{1} \succ s_{4} \succ s_{2} \succ s_{3}$; while the ranking about criterion 3 is $s_{1} \succ s_{2} \succ s_{4} \succ s_{3}$. Although the rankings about the three about criteria are not the same, it is evident that the optimal supplier for the three experts is $s_{1}$. 


\subsection{Optimization ordering model for IFPRs under the priority of utility}

Plugging $R^{(1)}, R^{(2)}$, and $R^{(3)}$ into Models (11) and (12), and establishing optimization ordering models for the membership and non-membership IVFPRs under the priority of utility, yields the optimal solutions listed in Table 5 .

Table 5: Optimal solutions to ordering model for IFPRs under the priority of utility

\begin{tabular}{|c|c|c|c|c|c|c|c|c|c|c|c|c|}
\hline \multirow{4}{*}{$R^{(1)}$} & $\omega_{1}^{A}$ & $\omega_{2}^{A}$ & $\omega_{3}^{A}$ & $\omega_{4}^{A}$ & $\varepsilon_{12}^{A}$ & $\varepsilon_{13}^{A}$ & $\varepsilon_{14}^{A}$ & $\varepsilon_{23}^{A}$ & $\varepsilon_{24}^{A}$ & $\varepsilon_{34}^{A}$ & $\lambda_{A}^{(1)}$ & $D_{A}^{(1)}$ \\
\hline & 0.5577 & 0.1593 & 0.0440 & 0.2390 & 0.0222 & 0.0269 & 0 & 0.0162 & 0 & 0.2447 & 1 & -1 \\
\hline & $\omega_{1}^{B}$ & $\omega_{2}^{B}$ & $\omega_{3}^{B}$ & $\omega_{4}^{B}$ & $\varepsilon_{12}^{B}$ & $\varepsilon_{13}^{B}$ & $\varepsilon_{14}^{B}$ & $\varepsilon_{23}^{B}$ & $\varepsilon_{24}^{B}$ & $\varepsilon_{34}^{B}$ & $\lambda_{B}^{(1)}$ & $D_{B}^{(1)}$ \\
\hline & 0 & 0 & 0 & 1 & 0.5000 & 0.3000 & 0.5000 & 0.4000 & 0.7000 & 0.7000 & 1 & -1 \\
\hline \multirow{4}{*}{$R^{(2)}$} & $\omega_{1}^{A}$ & $\omega_{2}^{A}$ & $\omega_{3}^{A}$ & $\omega_{4}^{A}$ & $\varepsilon_{12}^{A}$ & $\varepsilon_{13}^{A}$ & $\varepsilon_{14}^{A}$ & $\varepsilon_{23}^{A}$ & $\varepsilon_{24}^{A}$ & $\varepsilon_{34}^{A}$ & $\lambda_{A}^{(2)}$ & $D_{A}^{(2)}$ \\
\hline & 0.8757 & 0.0973 & 0.0108 & 0.0162 & 0 & 0.1878 & 0.2818 & 0 & 0.5571 & 0 & 1 & -1 \\
\hline & $\omega_{1}^{B}$ & $\omega_{2}^{B}$ & $\omega_{3}^{B}$ & $\omega_{4}^{B}$ & $\varepsilon_{12}^{B}$ & $\varepsilon_{13}^{B}$ & $\varepsilon_{14}^{B}$ & $\varepsilon_{23}^{B}$ & $\varepsilon_{24}^{B}$ & $\varepsilon_{34}^{B}$ & $\lambda_{B}^{(2)}$ & $D_{B}^{(2)}$ \\
\hline & 0.2154 & 0.3231 & 0.3231 & 0.1385 & 0 & 0.2000 & 0.2087 & 0 & 0 & 0.1000 & 1 & -1 \\
\hline \multirow{4}{*}{$R^{(3)}$} & $\omega_{1}^{A}$ & $\omega_{2}^{A}$ & $\omega_{3}^{A}$ & $\omega_{4}^{A}$ & $\varepsilon_{12}^{A}$ & $\varepsilon_{13}^{A}$ & $\varepsilon_{14}^{A}$ & $\varepsilon_{23}^{A}$ & $\varepsilon_{24}^{A}$ & $\varepsilon_{34}^{A}$ & $\lambda_{A}^{(3)}$ & $D_{A}^{(3)}$ \\
\hline & 0.6207 & 0.1552 & 0.0689 & 0.1552 & 0 & 0 & 0 & 0.2077 & 0.2000 & 0.3923 & 1 & -1 \\
\hline & $\omega_{1}^{B}$ & $\omega_{2}^{B}$ & $\omega_{3}^{B}$ & $\omega_{4}^{B}$ & $\varepsilon_{12}^{B}$ & $\varepsilon_{13}^{B}$ & $\varepsilon_{14}^{B}$ & $\varepsilon_{23}^{B}$ & $\varepsilon_{24}^{B}$ & $\varepsilon_{34}^{B}$ & $\lambda_{B}^{(3)}$ & $D_{B}^{(3)}$ \\
\hline & 0.1951 & 0.2927 & 0.4390 & 0.0732 & 0 & 0.1077 & 0.4273 & 0 & 0 & 0.0571 & 1 & -1 \\
\hline
\end{tabular}

Table 5 indicates that utility maximisation is achieved under the priority of utility in all cases: $\lambda_{A}^{(1)}=\lambda_{B}^{(1)}=\lambda_{A}^{(2)}=\lambda_{B}^{(2)}=\lambda_{A}^{(3)}=\lambda_{B}^{(3)}=1$. In addition, analytic results in Table 5 state that the ranking about criterion 1 is $s_{1} \succ s_{4} \succ s_{2} \succ s_{3}$, the ranking about criterion 2 is $s_{1} \succ s_{2} \succ s_{4} \succ s_{3}$, while the ranking about criterion 3 is $s_{1} \succ s_{2} \equiv s_{4} \succ s_{3}$. Although rankings about the three criteria are not the same, it is again evident that their optimal supplier is $s_{1}$.

\subsection{Optimization ordering model for IFPRs under the priority of deviation}

Similarly, Plugging $R^{(1)}, R^{(2)}$, and $R^{(3)}$ into Model (13) and (14), and establishing optimization ordering models for the membership and non-membership degree IVFPRs under the priority of deviation, yields the optimal solutions listed in Table 6 .

Analytic results in Table 6, which are the same as Table 4, provide us with evidence for the correctness of Corollary 1.

\subsection{Comparison analysis and the advantages of the proposed approach}

In this subsection, numerical examples of $[32,33]$ are plugged into Model (3) constructed in this paper, devoting to comparison analysis and advantage analysis. Without loss of generality, we can assume that all examples satisfy utility functions with right-skewed parabolic type, and the importance of three criteria is $\left(\frac{1}{3}, \frac{1}{3}, \frac{1}{3}\right)^{T}$.

Combining with analysis of methods in $[32,33]$ and comparison results in Table 7 , the proposed method of this paper has the following advantages: 
Table 6: Optimal solutions to the ordering model for IFPRs under the priority of deviation

\begin{tabular}{|c|c|c|c|c|c|c|c|c|c|c|c|c|}
\hline \multirow{4}{*}{$R^{(1)}$} & $\omega_{1}^{A}$ & $\omega_{2}^{A}$ & $\omega_{3}^{A}$ & $\omega_{4}^{A}$ & $\varepsilon_{12}^{A}$ & $\varepsilon_{13}^{A}$ & $\varepsilon_{14}^{A}$ & $\varepsilon_{23}^{A}$ & $\varepsilon_{24}^{A}$ & $\varepsilon_{34}^{A}$ & $\lambda_{A}^{(1)}$ & $W_{A}^{(1)}$ \\
\hline & 0.3873 & 0.2308 & 0.1065 & 0.2754 & 0 & 0 & 0 & 0 & 0.1138 & 0.0634 & 0.1779 & 0.1772 \\
\hline & $\omega_{1}^{B}$ & $\omega_{2}^{B}$ & $\omega_{3}^{B}$ & $\omega_{4}^{B}$ & $\varepsilon_{12}^{B}$ & $\varepsilon_{13}^{B}$ & $\varepsilon_{14}^{B}$ & $\varepsilon_{23}^{B}$ & $\varepsilon_{24}^{B}$ & $\varepsilon_{34}^{B}$ & $\lambda_{B}^{(1)}$ & $W_{B}^{(1)}$ \\
\hline & 0.1295 & 0.2173 & 0.4711 & 0.1821 & 0 & 0 & 0 & 0 & 0.1138 & 0.0634 & 0.3343 & 0.1772 \\
\hline \multirow{4}{*}{$R^{(2)}$} & $\omega_{1}^{A}$ & $\omega_{2}^{A}$ & $\omega_{3}^{A}$ & $\omega_{4}^{A}$ & $\varepsilon_{12}^{A}$ & $\varepsilon_{13}^{A}$ & $\varepsilon_{14}^{A}$ & $\varepsilon_{23}^{A}$ & $\varepsilon_{24}^{A}$ & $\varepsilon_{34}^{A}$ & $\lambda_{A}^{(2)}$ & $W_{A}^{(2)}$ \\
\hline & 0.4426 & 0.1903 & 0.1106 & 0.2565 & 0 & 0 & 0 & 0 & 0.1260 & 0.0986 & 0.1095 & 0.2246 \\
\hline & $\omega_{1}^{B}$ & $\omega_{2}^{B}$ & $\omega_{3}^{B}$ & $\omega_{4}^{B}$ & $\varepsilon_{12}^{B}$ & $\varepsilon_{13}^{B}$ & $\varepsilon_{14}^{B}$ & $\varepsilon_{23}^{B}$ & $\varepsilon_{24}^{B}$ & $\varepsilon_{34}^{B}$ & $\lambda_{B}^{(2)}$ & $W_{B}^{(2)}$ \\
\hline & 0.1105 & 0.2569 & 0.4420 & 0.1906 & 0 & 0 & 0 & 0 & 0.1260 & 0.0986 & 0.4476 & 0.2246 \\
\hline \multirow{4}{*}{$R^{(3)}$} & $\omega_{1}^{A}$ & $\omega_{2}^{A}$ & $\omega_{3}^{A}$ & $\omega_{4}^{A}$ & $\varepsilon_{12}^{A}$ & $\varepsilon_{13}^{A}$ & $\varepsilon_{14}^{A}$ & $\varepsilon_{23}^{A}$ & $\varepsilon_{24}^{A}$ & $\varepsilon_{34}^{A}$ & $\lambda_{A}^{(3)}$ & $W_{A}^{(3)}$ \\
\hline & 0.4925 & 0.2264 & 0.1000 & 0.1811 & 0.0228 & 0 & 0 & 0 & 0.3244 & 0 & 0.0972 & 0.3472 \\
\hline & $\omega_{1}^{B}$ & $\omega_{2}^{B}$ & $\omega_{3}^{B}$ & $\omega_{4}^{B}$ & $\varepsilon_{12}^{B}$ & $\varepsilon_{13}^{B}$ & $\varepsilon_{14}^{B}$ & $\varepsilon_{23}^{B}$ & $\varepsilon_{24}^{B}$ & $\varepsilon_{34}^{B}$ & $\lambda_{B}^{(3)}$ & $W_{B}^{(3)}$ \\
\hline & 0.0924 & 0.2011 & 0.4551 & 0.2514 & 0.0228 & 0 & 0 & 0 & 0.3244 & 0 & 0.4737 & 0.3472 \\
\hline
\end{tabular}

Table 7: Comparison results between other researches and Models constructed by this paper

\begin{tabular}{ccc}
\hline Methods & $\omega_{i}^{* T}$ or Score crT $_{i}^{* T}$ & Optimal Ranking \\
\hline$[32]$ & Score $_{i}^{* T}=(0.5426,0.5618,0.6025,0.6367)^{T}$ & $s_{4} \succ s_{3} \succ s_{2} \succ s_{1}$ \\
Model $(3)$ & $\omega_{i}^{* T}=(0.4339,0.1205,0.2109,0.1734)^{T}$ & $s_{1} \succ s_{3} \succ s_{4} \succ s_{2}$ \\
\hline$[33]$ & $\omega_{i}^{* T}=(0.0788,-0.4968,-0.7219,-0.8932)^{T}$ & $s_{1} \succ s_{2} \succ s_{3} \succ s_{4}$ \\
Model (3) & $\omega_{i}^{* T}=(0.3535,0.2849,0.2162,0.1122)^{T}$ & $s_{1} \succ s_{2} \succ s_{3} \succ s_{4}$ \\
\hline Model (11) & $\omega_{i}^{* T}=(0.6847,0.1373,0,0.1368)^{T}$ & $s_{1} \succ s_{2} \succ s_{4} \succ s_{3}$ \\
Model (3) & $\omega_{i}^{* T}=(0.4408,0.2158,0.1057,0.2377)^{T}$ & $s_{1} \succ s_{4} \succ s_{2} \succ s_{3}$ \\
\hline Model (13) & $\omega_{i}^{* T}=(0.4408,0.2158,0.1057,0.2377)^{T}$ & $s_{1} \succ s_{4} \succ s_{2} \succ s_{3}$ \\
Model (3) & $\omega_{i}^{* T}=(0.4408,0.2158,0.1057,0.2377)^{T}$ & $s_{1} \succ s_{4} \succ s_{2} \succ s_{3}$ \\
\hline
\end{tabular}

- [32] developed an algorithm to improve the additive consistency degree of IFPRs, while this paper concentrates on GDM with multiplicative consistent IVFPRs by model construction. Hence, with the same purpose of consistency, the proposed models are more flexible and comprehensive.

- The optimal ranking of alternatives depends on the normalized intuitionistic fuzzy weights in [33], apart from that, this paper introduces DMs' utility into GDM problems, which implies our method has a wide range of applications than [33].

- Model (11) gives priority to utility maximisation, and weakens consistency degree, which might be more suitable for some scenarios. The combination of Model (11) and Model (13) (same as Model (3)) satisfies various GDM problems. 


\section{Conclusions}

The ranking problem has always been a hot topic in IFPR research. The conventional method usually transforms IFPRs into the equivalent membership and non-membership IVFPRs. Then, the optimal solution for ranking is obtained by the operations of intervals. This often causes the amplification and reduction of numerical values, and even leads to the distortion of decision results. Although the DM only gives an interval judgment to a problem, their utility can reflect the DM's preference for the value of the judgment interval. The paper introduces the DM's non-linear utility preference (such as a parabolic utility function, S-shaped utility function, and HARA utility function), and takes utility preference as the value of interval preference. Subsequently, the ranking models for the membership and non-membership IVFPRs based on non-linear utility functions are constructed, respectively. In the two proposed models, a utility function is adopted to fit the true value of DM's uncertain judgment, and the objective function minimises the distance deviation between the ideal judgment (obeying multiplicative consistency) and the actual judgment (represented by a utility function). In addition, the optimal ranking relationship between two proposed models is investigated.

The main contributions of this article can be summarised as follows.

- Utility functions (including parabolic utility functions, S-shaped utility functions, and HARA utility functions) have been utilised to obtain the true value of the degree of membership (nonmembership) interval judgment, which are favourable for acquiring the true judgment information from the DM's uncertain judgment.

- As per the equivalent IVFPRs (the membership and non-membership IVFPRs) to given IFPRs, the ranking for IFPRs can obtained. The study has also proved that a reversal connection exists between the ranking for membership and non-membership IVFPRs.

- To minimise the distance deviation between the ideal judgment (obeying multiplicative consistency) and the actual judgment (represented by utility function), the optimization ordering model for IFPRs based on utility function has been constructed.

- By introducing a non-Archimedean infinitesimal into the proposed models, the optimization ordering models for IFPRs under the priority of utility and distance deviation have been established to realise the priority solution of multi-objective programming meeting the different priority objectives of alternatives ranking.

\section{Acknowledgments}

This research is partially supported by the National Natural Science Foundation of China (Grant \#: 71171115, 71571104), the Reform Foundation of Postgraduate Education and Teaching in Jiangsu 
Province (Grant \#: JGKT10034), a Six Talent Peaks Project in Jiangsu Province (Grant \#: 2014JY-014), Top-notch Academic Programs Project of Jiangsu Higher Education Institutions, and the Postgraduate Research \& Practice Innovation Program of Jiangsu Province (KYCX17_0904).

\section{References}

[1] Z.W. Gong, X.X. Xu, H.H. Zhang, U.A. Ozturk, E. Herrera-Viedma, C. Xu, The consensus models with interval preference opinions and their economic interpretation, Omega 55 (2015) 81-90.

[2] G. Büyüközkan, S. Güleryüz, Evaluation of renewable energy resources in turkey using an integrated mcdm approach with linguistic interval fuzzy preference relations, Energy 123 (2017) 149-163.

[3] S. Wan, F. Wang, J. Dong, A group decision making method with interval valued fuzzy preference relation based on the geometric consistency, Information Fusion 40 (2018) 87-100.

[4] J. Wu, F. Chiclana, A risk attitudinal ranking method for interval-valued intuitionistic fuzzy numbers based on novel score and accuracy expected functions, Applied Soft Computing 22 (2014) 272-286.

[5] N. Zhang, Z.W. Gong, F. Chiclana, Minimum cost consensus models based on random opinions, Expert Systems with Applications 89 (2017) 149-159.

[6] F. Meng, J. Tang, Z. Xu, A 0-1 mixed programming model based method for group decision making with intuitionistic fuzzy preference relations, Computers \& Industrial Engineering 112 (2017) 289-304.

[7] S. Wan, F. Wang, J. Dong, Additive consistent interval-valued atanassov intuitionistic fuzzy preference relation and likelihood comparison algorithm based group decision making, European Journal of Operational Research 263 (2017) 571-582.

[8] Z.W. Gong, X.X. Xu, Y.J. Yang, Y. Zhou, H.H. Zhang, The Spherical Distance for Intuitionistic Fuzzy Sets and lts Application in Decision Analysis, Technological and Economic Development of Economy 22 (2016) 393-415.

[9] L.H. Wang, Z.W. Gong, Priority of a hesitant fuzzy linguistic preference relation with a normal distribution in meteorological disaster risk assessment, International Journal of Environmental Research \& Public Health 14(10) (2017) 1203.

[10] Z. Ren, Z. Xu, H. Wang, Dual hesitant fuzzy VIKOR method for multi-criteria group decision making based on fuzzy measure and new comparison method, Information Sciences 388 (2017) $1-16$. 
[11] W. Zhou, Z.S. Xu, Group consistency and group decision making under uncertain probabilistic hesitant fuzzy preference environment, Information Sciences 414 (2017) 276-288.

[12] F. Herrera, E. Herrera-Viedma, J.L. Verdegay, Choice processes for non-homogeneous group decision making in linguistic setting, Fuzzy Sets and Systems 94 (1998) 287-308.

[13] M. Delgado, F. Herrera, E. Herrera-Viedma, L. Martínez, Combining numerical and linguistic information in group decision making, Information Sciences 107 (1998) 177-194.

[14] F. Herrera, L. Martínez, P.J. Sánchez, Managing non-homogeneous information in group decision making. European Journal of Operational Research 166 (2005) 115-132.

[15] J. Wu, F. Chiclana, Visual information feedback mechanism and attitudinal prioritisation method for group decision making with triangular fuzzy complementary preference relations, Information Sciences 279 (2014) 716-734.

[16] S.M. Chen, S.H. Cheng, T.E. Lin, Group decision making systems using group recommendations based on interval fuzzy preference relations and consistency matrices, Information Sciences 298 (2015) 555-567.

[17] F. Meng, X. Chen, M. Zhu, J. Lin, Two new methods for deriving the priority vector from interval multiplicative preference relations, Information Fusion 26 (2015) 122-135.

[18] F. Meng, X. Chen, Y. Zhang, Consistency-based linear programming models for generating the priority vector from interval fuzzy preference relations, Applied Soft Computing 41 (2016) 247264.

[19] A. Khalid, I. Beg, Incomplete interval valued fuzzy preference relations, Information Sciences 348 (2016) 15-24.

[20] X. Peng, Y. Yang, Algorithms for interval-valued fuzzy soft sets in stochastic multi-criteria decision making based on regret theory and prospect theory with combined weight, Applied Soft Computing 54 (2017) 415-430.

[21] J. Wu, F. Chiclana, H. Liao, Isomorphic multiplicative transitivity for intuitionistic and intervalvalued fuzzy preference relations and its application in deriving their priority vectors, IEEE Transactions on Fuzzy Systems 26 (1) (2018) 193-202.

[22] J. Wu, F. Chiclana, Non-dominance and attitudinal prioritisation methods for intuitionistic and interval-valued intuitionistic fuzzy preference relations, Expert Systems with Applications 39 (2012) 13409-13416. 
[23] S. Zeng, W. Su, L. Sun, A method based on similarity measures for interactive group decisionmaking with intuitionistic fuzzy preference relations, Applied Mathematical Modelling 37 (10-11) (2013) 6909-6917.

[24] S.P. Wan, F. Wang, J.Y. Dong, A preference degree for intuitionistic fuzzy values and application to multi-attribute group decision making, Information Sciences 370-371 (2016) 127-146.

[25] Z.J. Wang, Derivation of intuitionistic fuzzy weights based on intuitionistic fuzzy preference relations, Applied Mathematical Modelling 37 (2013) 6377-6388.

[26] Q. Mou, Z. Xu, H. Liao, A graph based group decision making approach with intuitionistic fuzzy preference relations, Computers \& Industrial Engineering 110 (2017) 138-150.

[27] J. Wu, F. Chiclana, Multiplicative consistency of intuitionistic reciprocal preference relations and its application to missing values estimation and consensus building, Knowledge-Based Systems 71 (2014) 187-200.

[28] H. Behret, Group decision making with intuitionistic fuzzy preference relations, Knowledge-Based Systems 70 (2014) 33-43.

[29] H.C. Liao, Z.S. Xu, Consistency of the fused intuitionistic fuzzy preference relation in group intuitionistic fuzzy analytic hierarchy process, Applied Soft Computing 35 (2015) 812-826.

[30] S.P. Wan, G.L. Xu, J.Y. Dong, A novel method for group decision making with interval-valued atanassov intuitionistic fuzzy preference relations, Information Sciences 372 (2016) 53-71.

[31] G.L. Xu, S.P. Wan, F. Wang, J.Y. Dong, Y.F. Zeng, Mathematical programming methods for consistency and consensus in group decision making with intuitionistic fuzzy preference relations, Knowledge-Based Systems 98 (2016) 30-43.

[32] J. Chu, X. Liu, Y. Wang, K.S. Chin, A group decision making model considering both the additive consistency and group consensus of intuitionistic fuzzy preference relations, Computers \& Industrial Engineering 101 (2016) 227-242.

[33] F. Jin, Z. Ni, H. Chen, Y. Li, Approaches to group decision making with intuitionistic fuzzy preference relations based on multiplicative consistency, Knowledge-Based Systems 97 (2016) 48-59.

[34] H.S. Houthakker, Revealed preference and the utility function, Economica 17 (66) (1950) 159-174.

[35] R.C. Merton, Lifetime portfolio selection under uncertainty: The continuous-time case, The review of Economics and Statistics (1969) 247-257. 
[36] R.C. Merton, Optimum consumption and portfolio rules in a continuous-time model, Journal of economic theory 3 (4) (1971) 373-413.

[37] P.C. Fishburn, Utility theory for decision making, New York: Wiley (1970).

[38] K. Borch, Decision rules depending on the probability of ruin, Oxford Economic Papers 20 (1) (1968) 1-10.

[39] E. Castagnoli, M.L. Calzi, Expected utility without utility, Theory and Decision 41 (3) (1996) 281-301.

[40] R.F. Bordley, C.W. Kirkwood, Multiattribute preference analysis with performance targets, Operations Research 52 (6) (2004) 823-835.

[41] I. Tsetlin, R.L. Winkler, Decision making with multiattribute performance targets: the impact of changes in performance and target distributions, Operations Research 55 (2) (2007) 226-233.

[42] I. Durbach, On the estimation of a satisficing model of choice using stochastic multicriteria acceptability analysis, Omega 37 (3) (2009) 497-509.

[43] C.T. Chang, Multi-choice goal programming with utility functions, European Journal of Operational Research 215 (2011) 439-445.

[44] C.T. Chang, Fuzzy linearization strategy for multiple objective linear fractional programming with binary utility functions, Computers \& Industrial Engineering 112 (2017) 437-446.

[45] R.M. Musal, R. Soyerb, S.A. Kharroubi, Estimating the population utility function: a parametric bayesian approach, European Journal of Operational Research 218 (2012) 538-547.

[46] E. Çanakoğlu, Portfolio selection in stochastic markets with hara utility functions, European Journal of Operational Research 201 (2010) 520-536.

[47] E.J. Jung, J.H. Kim, Optimal investment strategies for the HARA utility under the constant elasticity of variance model, Insurance: Mathematics and Economics 51 (3) (2012) 667-673.

[48] J.H. Choi, H. Ahn, I. Han, Utility-based double auction mechanism using genetic algorithms, Expert systems with applications 34 (1) (2008) 150-158.

[49] F.J. André, L. Riesgo, A non-interactive elicitation method for non-linear multiattribute utility functions: Theory and application to agricultural economics, European Journal of Operational Research 181 (2) (2007) 793-807.

[50] H.P. Ho, C.T. Chang, C.Y. Ku, House selection via the internet by considering homebuyers risk attitudes with s-shaped utility functions, European Journal of Operational Research 241 (2015) 188-201. 
[51] M. Kang, L.S. Ye, Advantageous redistribution with three smooth ces utility functions, Journal of Mathematical Economics 67 (2016) 171-180.

[52] J. Breinbjerg, Equilibrium arrival times to queues with general service times and non-linear utility functions, European Journal of Operational Research 261 (2017) 595-605.

[53] D. Kahneman, A. Tversky, On the interpretation of intuitive probability: a reply to jonathan cohen, Cognition 7 (1979) 409-411.

[54] B. Feng, F. Lai, Multi-attribute group decision making with aspirations: a case study, Omega 44 (1994) 136-147.

[55] M. Grasselli, A stability result for the HARA class with stochastic interest rates, Insurance: Mathematics and Economic 33 (2003) 611-627.

[56] J. C. Bezdek, B. Spillman, R. Spillman, A fuzzy relation space for group decision theory, Fuzzy Sets and Systems 1 (4) (1978) 255-268.

[57] H. Nurmi, Approaches to collective decision making with fuzzy preference relations, Fuzzy Sets and Systems 6 (3) (1981) 249-259.

[58] F. Chiclana, E. Herrera-Viedma, S. Alonso, F. Herrera, Cardinal consistency of reciprocal preference relations: a characterization of multiplicative transitivity, IEEE Transactions on Fuzzy Systems 17 (1) (2009) 14-23.

[59] J. Wu, F. Chiclana, A social network analysis trust-consensus based approach to group decisionmaking problems with interval-valued fuzzy reciprocal preference relations, Knowledge-Based Systems 59 (2014) 97-107.

[60] R. Urena, F. Chiclana, H. Fujita, E. Herrera-Viedma, Confidence-consistency driven group decision making approach with incomplete reciprocal intuitionistic preference relations, KnowledgeBased Systems 89 (2015) 86-96.

[61] K.T. Atanassov, Intuitionistic fuzzy sets, Fuzzy Sets and Systems (1986) 89-97.

[62] Z.W. Gong, L.S. Li, J. Cao, F.X. Zhou, On additive consistent properties of the intuitionistic fuzzy preference relation, International Journal of Information Technology \& Decision Making 9 (2010) 1009-1025.

[63] A. Charnes, Optimality and degeneracy in linear programming, Econometrica 20 (1952) 160-170.

[64] Z.S. Xu, H.C. Liao, Intuitionistic fuzzy analytic hierarchy process, IEEE Transactions on Fuzzy Systems 22 (2014) 749-761. 
[65] H. Liao, Z. Xu, X.J. Zeng, D.L. Xu, An enhanced consensus reaching process in group decision making with intuitionistic fuzzy preference relations, Information Sciences 329 (2015) 274-286. 